\title{
Optimization of Process Parameters in Dissimilar Joining between SAPH 440 steel with 6061 aluminum alloy by MIG Brazing
}

\section{Research Article}

Volume 2 Issue 1- 2021

\begin{abstract}
Author Details
Niwat Mookam ${ }^{1 \star}$, Tavee Madsa ${ }^{2}$, Shahrul Hisyam bin Marwan ${ }^{3}$ and Mohd Faizul Idham bin Mohd Zulkipli ${ }^{3}$

${ }^{1}$ Department of Industrial and Production Engineering, Rajamangala University of Technology Rattanakosin Wang Klai Kangwon Campus, Thailand

${ }^{2}$ Department of Industrial Engineering Technology, Rajamangala University of Technology Rattanakosin Wang Klai Kangwon Campus, Thailand

${ }^{3}$ Faculty of Mechanical Engineering, Universiti Teknologi MARA, Terengganu Branch, Bukit Besi Campus, Malaysia
\end{abstract}

*Corresponding author

Department of Industrial and Production Engineering, Faculty of Industry and Technology, Rajamangala University of Technology Rattanakosin Wang Klai Kangwon Campus, Prachuapkhirikhan 77110, Thailand

Article History

Received: February 01, 2021 Accepted: February 03, 2021 Published: February 04, 2021

\begin{abstract}
The aim of this paper was to determine an optimal brazing condition for dissimilar joining between SAPH 440 steel with 6061 aluminum alloy. The brazing variables investigated in this study encompassed brazing speed, brazing current, wire feed rate, and torch distance and its angle. Taguchi technique was employed as the experimental strategy, and response optimizations on shear strength were performed using the $\mathrm{S} / \mathrm{N}$ ratio. Results indicated that brazing variables have given a significant effect on the shear strength behavior. The optimal brazing condition was at $540 \mathrm{~mm} / \mathrm{min}$ of brazing speed, $25 \mathrm{~A}$ of brazing current, $8 \mathrm{~m} / \mathrm{min}$ of wire feed rate, $3 \mathrm{~mm}$ of torch distance, and 80 degree of torch angle. Lastly, the maximum shear strength prediction of the optimal condition was $3810.50 \mathrm{~N}$. Confirmation tests on the optimal brazing condition were $3451.21 \mathrm{~N}$.
\end{abstract}

Keywords: Optimization, Process parameters, Dissimilar joining, Shear strength, MIG brazing, SAPH 440 steel, 6061 aluminum alloy

\section{Introduction}

The aluminum alloy and steel sheet are widely used for complex parts due to its high strength, good formability and lower density [1]. However, these dissimilar materials are difficult to be jointed together. This happens because of the dissimilar and different physical properties shown by the materials. Aluminum alloy has low melting temperature, high thermal conductivity and solubility, oxide formation and high solidification shrinkage, which is in contrast to steel properties [2]. Aluminum alloy is well-known can be easily oxidized at the higher temperature or elevated heat input of welding process. In general, the higher heat input of welding process affects the joint strength because it promotes intermetallic compound (IMC) formation and growth [3, 4]. The strength and the reliability of joints are related to the IMC layer thickness. The growth of the IMC can reduce mechanical strength of the joint.

Normally, MIG/MAG welding machine have been utilized in automotive manufacturing industries due to its easily controllable joining process for sheets metal. Thus, MIG is applicable application to be applied in joining of aluminum and steel alloys because it presents the excellent heat input controllable. In addition, the spreadability of filler metal liquid on the weld surface contributes to the improvement of mechanical properties of dissimilar sheet join [4,5]. In such applications, welding and joining process have to involve which the optimization parameters setting would lead to a safety and joint ability under working conditions. In general, there are several approaches can be used in determining the optimal parameter such as mathematical and statistical approaches. It is well known that the Taguchi method is used to determine the factors affecting the out most, with a minimum amount of experimentation, thus saving time and resources for industry [6,7]. Therefore, this paper determined an optimal brazing condition of dissimilar joining between SAPH 440 steel and 6061 aluminum alloy by MIG brazing. The brazing variables examined including brazing speed, brazing current, wire feed rate, torch distant, and torch angle. The response optimization on shear strength was investigated in this study. Taguchi method with the $\mathrm{S} / \mathrm{N}$ ratio was used to identify those optimal brazing condition. 


\section{Methods and Materials}

In this experiment, different steel sheets of SAPH 440 steel and 6061 aluminum alloy with $1.0 \mathrm{~mm}$ thickness respectively were brazed by MIG/MAG welding machine (Fronius, Vario Star 2500). The chemical composition of the steel and aluminum alloy in this study is shown in Table 1 and 2 respectively. The substrate sheet was cut in dimension of $10 \times 10 \mathrm{~cm}$ and the surface was chemically cleaned by acetone before brazing. In brazing process, the $\mathrm{Al}-12 \mathrm{Si}$ wt $\%$ filler metal with diameter of $1.0 \mathrm{~mm}$ and high-purity argon gas for weld protection were used. The brazed joint with $20 \mathrm{~mm}$ overlap length where the aluminum alloy and steel plates were at the top and bottom respectively. The brazed sheet schematic and geometric illustrations for such lap joint are shown in Figure 1. After brazing, the brazed samples were cut in dimension of 20x60 mm. A wire-EDM (Mitsubishi, FA20S Advance) was used in cutting the samples. The lap shear testing was carried out using a universal testing machine (Zwick, Z020).

Table 1: Chemical composition of SAPH 440 steel.

\begin{tabular}{|c|c|c|c|c|c|c|}
\hline $\mathbf{C}$ & $\mathbf{S i}$ & $\mathbf{M n}$ & $\mathbf{P}$ & $\mathbf{S}$ & $\mathbf{A l}$ & $\mathbf{F e}$ \\
\hline 0.21 & 0.3 & 1.5 & 0.03 & 0.025 & 0.01 & Bal. \\
\hline
\end{tabular}

Table 2: Chemical composition of 6061 aluminum alloy.

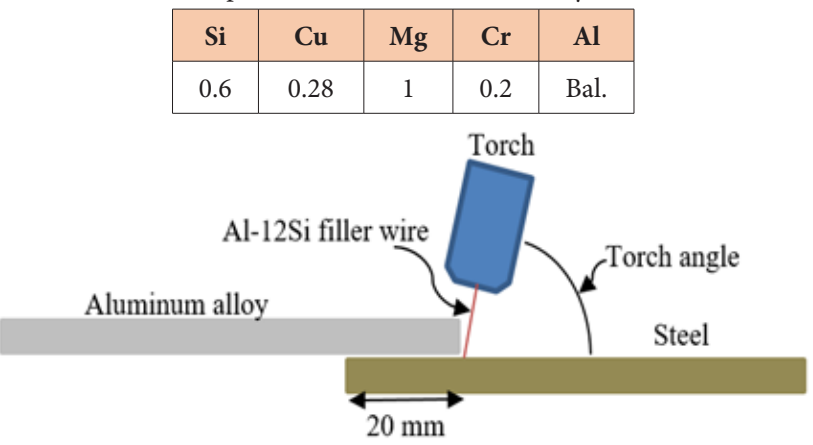

Figure 1: Geometry and schematic illustration of brazed joints.

In this experimentation, the controlled variables and levels selected are shown in Table 3. The control factors were chosen based on past researches and the user manual guide of MIG/MAG welding machine. The experimental design was according to an L27 orthogonal array based on the Taguchi method. The results were analyzed by employing main effects, and the signal-to-noise ratio ( $\mathrm{S} / \mathrm{N}$ ratio) analyses. The optimal levels were chosen with the largest $\mathrm{S} / \mathrm{N}$ ratio, and the predicted shear strength from the optimal brazing condition was performed using Minitab software. Finally, a confirmation test was carried out to compare the experimental results with the estimated results.

Table 3: Process parameters and levels.

\begin{tabular}{|c|c|c|c|c|c|}
\hline \multirow{2}{*}{ Control variables } & \multirow{2}{*}{ Symbols } & \multirow{2}{*}{ Units } & \multicolumn{3}{|c|}{ Levels } \\
\cline { 4 - 6 } & & & Low & Medium & High \\
\hline Brazing speed & (A) & $\mathrm{mm} / \mathrm{min}$ & 540 & 850 & 1,500 \\
\hline Brazing current & (B) & $\mathrm{A}$ & 25 & 50 & 75 \\
\hline Wire feed rate & (C) & $\mathrm{m} / \mathrm{min}$ & 4 & 6 & 8 \\
\hline Torch distant & (D) & $\mathrm{mm}$ & 3 & 4 & 5 \\
\hline Torch angle & (E) & degree & 70 & 80 & 90 \\
\hline
\end{tabular}

\section{Results and Discussions}

The shear strength result for each condition is tabulated in Table 4. The results were analyzed by employing main effects, and the signal-tonoise ratio $(\mathrm{S} / \mathrm{N})$ analyses. The response of $\mathrm{S} / \mathrm{N}$ was displayed in Table 5 , and the plots of each response variables to the predictive factors were shown in Figure 2. As a result, the brazing speed (A), brazing current (B) and torch distance (D), was set at low level condition improved the shear strength of the workpiece. In the other hand, higher value of wire feed rate $(\mathrm{C})$ and medium level of torch angle (E) are desirable to contribute good shear strength.

Table 4: Shear strength results of each conditions.

\begin{tabular}{|c|c|c|c|c|c|c|}
\hline No. & (A) & (B) & (C) & (D) & (E) & Shear strength $(\mathrm{N})$ \\
\hline 1 & 540 & 25 & 4 & 3 & 70 & 3075.73 \\
\hline 2 & 540 & 25 & 4 & 3 & 80 & 3285.61 \\
\hline 3 & 540 & 25 & 4 & 3 & 90 & 3441.27 \\
\hline 4 & 540 & 50 & 6 & 4 & 70 & 2449.64 \\
\hline 5 & 540 & 50 & 6 & 4 & 80 & 2329.35 \\
\hline 6 & 540 & 50 & 6 & 4 & 90 & 1799.66 \\
\hline 7 & 540 & 75 & 8 & 5 & 70 & 1728.28 \\
\hline 8 & 540 & 75 & 8 & 5 & 80 & 2346.04 \\
\hline 9 & 540 & 75 & 8 & 5 & 90 & 822.37 \\
\hline 10 & 850 & 25 & 6 & 5 & 70 & 2675.86 \\
\hline 11 & 850 & 25 & 6 & 5 & 80 & 2387.62 \\
\hline 12 & 850 & 25 & 6 & 5 & 90 & 1966.42 \\
\hline 13 & 850 & 50 & 8 & 3 & 70 & 2277.12 \\
\hline 14 & 850 & 50 & 8 & 3 & 80 & 2361.57 \\
\hline 15 & 850 & 50 & 8 & 3 & 90 & 2035.46 \\
\hline 16 & 850 & 75 & 4 & 4 & 70 & 815.73 \\
\hline 17 & 850 & 75 & 4 & 4 & 80 & 2073.8 \\
\hline 18 & 850 & 75 & 4 & 4 & 90 & 1154.27 \\
\hline 19 & 1500 & 25 & 8 & 4 & 70 & 2536.59 \\
\hline 20 & 1500 & 25 & 8 & 4 & 80 & 2484.07 \\
\hline 21 & 1500 & 25 & 8 & 4 & 90 & 2053.42 \\
\hline 22 & 1500 & 50 & 4 & 5 & 70 & 954.76 \\
\hline 23 & 1500 & 50 & 4 & 5 & 80 & 574.18 \\
\hline 24 & 1500 & 50 & 4 & 5 & 90 & 458.12 \\
\hline 25 & 1500 & 75 & 6 & 3 & 70 & 685.22 \\
\hline 26 & 1500 & 75 & 6 & 3 & 80 & 1305.34 \\
\hline 27 & 1500 & 75 & 6 & 3 & 90 & 1139.67 \\
\hline
\end{tabular}

Main Effects Plot (data means) for SN ratios

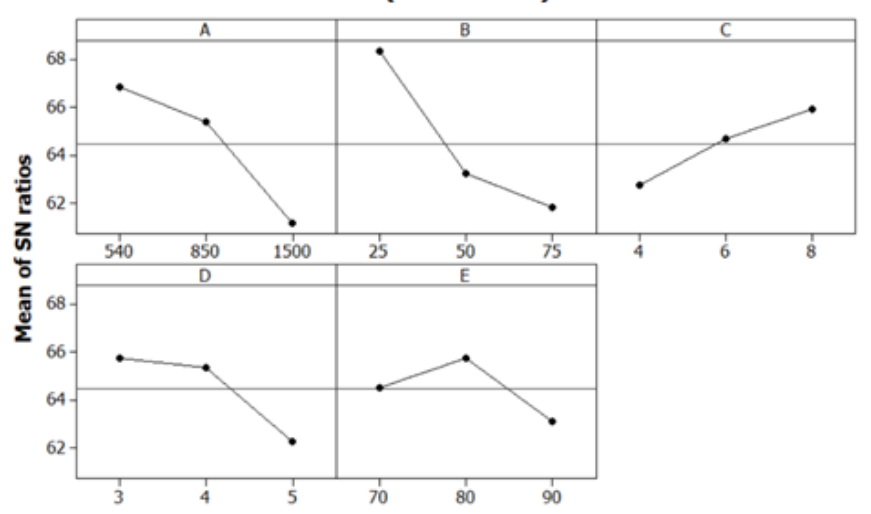

Figure 2: Main effect plot for $\mathrm{SN}$ ratios.

From the prediction, the optimal brazing conditions, i.e. $540 \mathrm{~mm} /$ min brazing speed, 25A brazing current, $8 \mathrm{~m} / \mathrm{min}$ wire feed rate, 3 $\mathrm{mm}$ brazing distant, and 80 degree brazing angle. The predicted shear strength of the brazing joint from the optimal brazing condition were $3810.50 \mathrm{~N}$. The samples were employed confirmation test, and the shear strength result was $3451.21 \mathrm{~N}$. Figure 4 indicates the brazing workpiece of optimal conditions. There are no defects e.g., pores and 
spatters on the surface of weld. The plot of shear strength of optimal brazing condition is shown in Figure 5.

Table 5: Signal to noise ratios of Control variables.

\begin{tabular}{|c|c|c|c|c|c|}
\hline \multicolumn{7}{|c|}{ Control variables } \\
\hline Level & A & B & C & D & E \\
\hline 1 & 66.84 & 68.34 & 62.74 & 65.75 & 64.51 \\
\hline 2 & 65.4 & 63.22 & 64.71 & 65.36 & 65.76 \\
\hline 3 & 61.16 & 61.83 & 65.94 & 62.27 & 63.12 \\
\hline Delta & 5.68 & 6.51 & 3.21 & 3.48 & 2.64 \\
\hline Rank & 2 & 1 & 4 & 3 & 5 \\
\hline
\end{tabular}

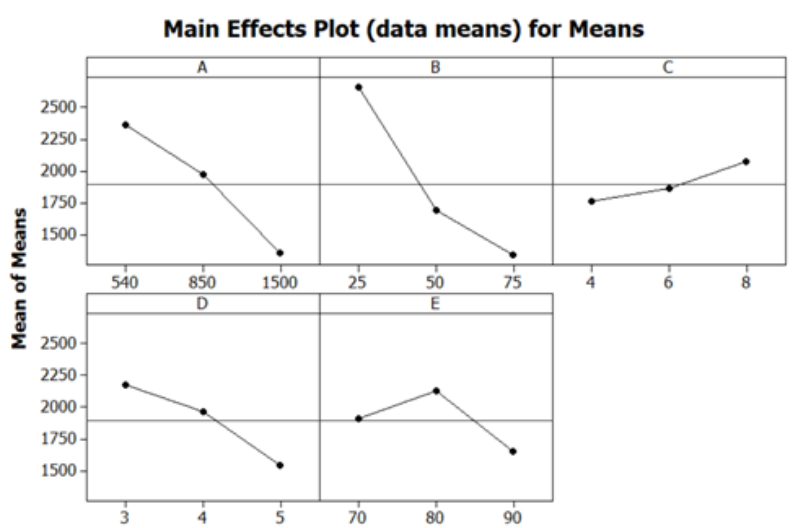

Figure 3: Main effect plot for response variable.

\section{Conclusion}

The optimization of process parameters for dissimilar Joint of SAPH 440 steel with 6061 aluminum alloy was performed in this study. The process variables investigated in this experiment included brazing speed, brazing current, wire feed rate, torch distant, and torch angle, while shear strength of the workpiece were the responses. Results showed that the optimization condition for maximum shear strength was obtained as brazing speed at $540 \mathrm{~mm} / \mathrm{min}$, brazing current at $25 \mathrm{~A}$, wire feed rate at $8 \mathrm{~m} / \mathrm{min}$, brazing distance at $3 \mathrm{~mm}$ and brazing angle at 80 degree. The shear strength of workpiece brazed with optimal brazing conditions was 3810.50 and $3451.21 \mathrm{~N}$. for the prediction and confirmation, respectively.

\section{Acknowledgement}

The authors would like to thank Rajamangala University of Technology Rattanakosin for the laboratory equipment support of this research.

\section{Conflicts of Interest}

The authors declare that they have no conflict of interest.

\section{References}

1. Danyang Dong, Yang Liu, Yuling Yang, Jinfeng Li, Min Ma, et al., (2014) Microstructure and dynamic tensile behavior of DP600 dual phase steel joint by laser welding. Materials Science \& Engineering A 594: 17-25.

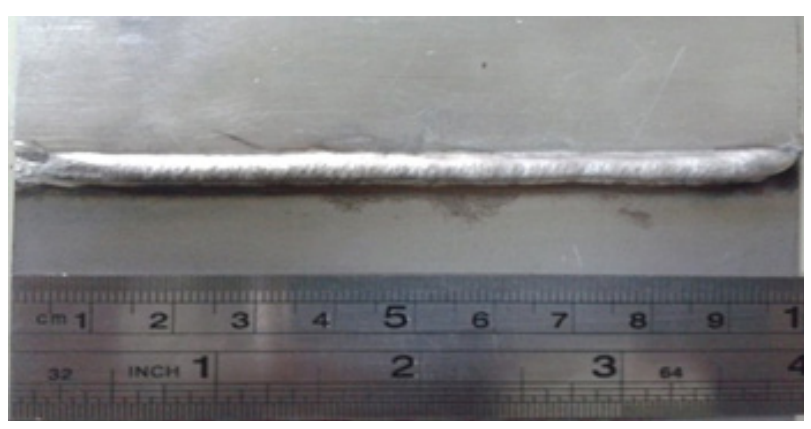

Figure 4: The samples of optimal brazing condition.

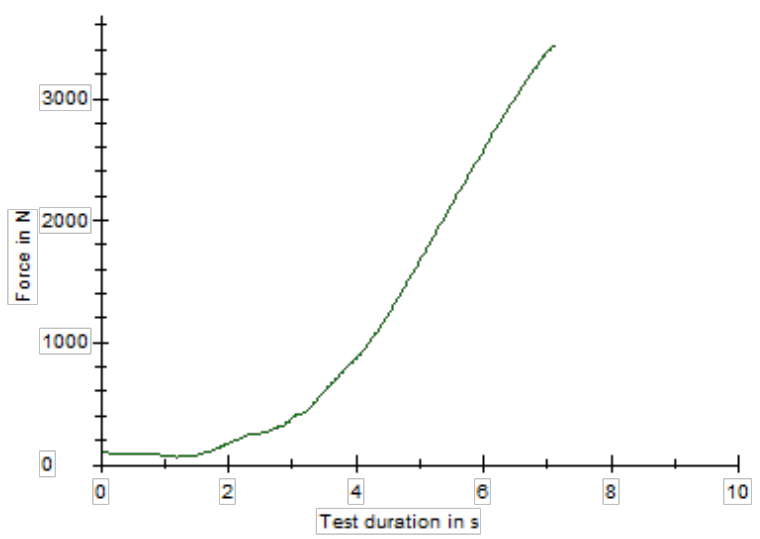

Figure 5: The shear strength of optimal brazing condition.

2. Sachindra J Doshi, A V Gohil, N D Mehta, S R Vaghasiya (2018) Challenges in Fusion Welding of Al alloy for Body in White. Materials Today: Proceedings 5: 6370-6375.

3. Yufeng Zhang, Jihua Huang, Zheng Ye, Zhi Cheng, Jian Yang. Et al., (2018) Influence of welding parameters on the IMCs and the mechanical properties of $\mathrm{Ti} / \mathrm{Al}$ butt joints welded by MIG/TIG double-sided arc welding-brazing. Journal of Alloys and Compounds 747: 764-771.

4. Zheng Ye, Jihua Huang, Wei Gao, Yufeng Zhang, Zhi Cheng, et al., (2017) Microstructure and mechanical properties of 5052 aluminum alloy/mild steel butt joint achieved by MIG-TIG double-sided arc welding-brazing. Materials and Design 123: 69-79.

5. Guoliang Qin, Yang Ji, Hong Ma, Zhiyong Ao (2017) Effect of modified flux on MIG arc brazing-fusion welding of aluminum alloy to steel butt joint. Journal of Materials Processing Technology 245: 115-121.

6. Amitesh Goswami, Jatinder Kumar (2014) Optimization in wire-cut EDM of Nimonic-80A using Taguchi's approach and utility concept. Engineering Science and Technology, an International Journal 17: 236246.

7. Vijay Verma, Ram Sajeevan (2015) Multi process parameter optimization of Diesinking EDM on Titanium alloy (Ti6Al4V) using Taguchi approach. Materials Today: Proceedings 2: 2581-2587. 